\title{
Genotyping of European isolates of methicillin- resistant Staphylococcus aureus by fluorescent amplified-fragment length polymorphism analysis (FAFLP) and pulsed-field gel electrophoresis (PFGE) typing
}

\author{
RUTH GRADY**, DOMINIQUE BLANC $\dagger$, PHILIPPE HAUSER $\dagger$ and JOHN STANLEY* \\ * Molecular Biology Unit, Central Public Health Laboratory, 61 Colindale Avenue, London and †Division \\ Autonome de Médecine Préventive Hospitalière, Centre Hospitalier Universitaire Vaudois, Lausanne, \\ Switzerland
}

\begin{abstract}
A representative panel of 50 European MRSA isolates was subjected to genotype analysis by fluorescent amplified-fragment length polymorphism (FAFLP) and by macrorestriction pulsed-field gel electrophoresis (PFGE). Each isolate had a unique profile with FAFLP. To model genetic relationships within the continuing MRSA epidemic, cluster analysis of FAFLP data was made, revealing nine clone complexes of MRSA. Most of these were also found by PFGE. A number of isolates had FAFLP profiles significantly different from others, and might represent emerging epidemic strains. FAFLP analysis proved particularly suitable for surveillance of the MRSA epidemic at national and international levels.
\end{abstract}

\section{Introduction}

Methicillin-resistant Staphylococcus aureus (MRSA) is a world-wide nosocomial pathogen, usually resistant to several antibiotics [1]. Currently, the leading effective therapy is intravenous vancomycin, which can be predicted to be effective before susceptibility testing of an MRSA isolate. In the UK in 1998, MRSA bacteraemia represented $32 \%$ of total $S$. aureus bacteraemia [2], the majority being acquired in hospital.

Epidemiological typing of isolates forms part of the effective infection control management of MRSA. Phage typing has been used to type clinical isolates of $S$. aureus for nearly 50 years [3]. Isolates are classified into broad 'phage groups' depending on their pattern of lysis by one or more of a set of internationally agreed phages. These groups can be subdivided into phage types, depending on particular

Received 20 Nov. 2000; accepted 8 Dec. 2000.

Corresponding author: Dr J. Stanley (e-mail: sevenwoods@ hotmail.com).

†Present address: Biomolecular Sciences, University of Manchester Institute of Science and Technology (UMIST), PO Box 88, Manchester M60 1QD. phage reactions. Although this simple phenotypic technique is easy to perform and convenient for large numbers of isolates, it has only limited discriminatory power. Moreover, in the USA, up to $20 \%$ of isolates are phage non-typable [4]. In England and Wales, 16 phage types of MRSA have affected more than one hospital, and are termed epidemic (EMRSA) [5]. In the 1990s, three of them (EMRSA-15, EMRSA-16 and EMRSA3) predominated in England and Wales, while other phage types receded in clinical significance [2].

A widely used molecular method for typing MRSA is pulsed-field gel electrophoresis (PFGE). In Continental Europe, $>20$ strains of EMRSA have been defined by PFGE [6]. PFGE is currently employed in the USA for integrated national surveillance of the enteric pathogen Escherichia coli $\mathrm{O} 157$ [7]. Some authors report that PFGE may produce unstable banding patterns with MRSA, generating problematic variation in interlaboratory studies [8]. The observed instability may be due to lysogenic integration of one or more phages in the $S$. aureus genome [9].

Fluorescent amplified-fragment length polymorphism (FAFLP) is based on selective amplification by PCR of a subset of restriction fragments from a digest of a whole bacterial genome. Typically, the DNA of an organism is digested with a restriction endonuclease 
with a six-base recognition sequence followed by digestion with a four-base recognition sequence endonuclease. Site-specific double-stranded adaptors are ligated to the ends of the fragments to generate primer binding sites. A subset of these restriction fragments amplified by PCR is separated on an automated sequencer. One of the site-specific primers is fluorescently labelled: only fragments cut with the corresponding enzyme are seen by the laser detector of the sequencer. Because a differentially labelled internal size standard is run in each gel track, all fragments are sized with precision $( \pm 1 \mathrm{bp})$. FAFLP thus offers a valuable new approach to high-resolution genotyping of MRSA. When applied to isolates of the UK epidemic phage type EMRSA-15, it generated reproducible DNA fingerprints, resolving isolates as members of a clone complex [10]. Similarly, isolates of all 16 UK epidemic phage types were resolved by FAFLP into five clone complexes [11]. In the present study, FAFLP was used to examine the genetic diversity of a range of European MRSA isolates, with a view to modelling the genetics of the continuing MRSA epidemic; the majority of isolates had been analysed previously by multiprimer RAPD-PCR typing [12] and they were also subjected to PFGE analysis.

\section{Materials and methods}

\section{Strains}

A representative panel of 50 methicillin-resistant isolates and reference strains of $S$. aureus from diverse European countries was examined (Table 1).

\section{Methods}

Bacteria were grown overnight on blood agar at $37^{\circ} \mathrm{C}$, in an aerobic atmosphere. Genomic DNA was isolated from plate cultures with lysostaphin-NaCl-cetyltrimethylammonium bromide [10]. The endonucleases EcoRI and MseI were used to digest c. $500 \mathrm{ng}$ of genomic DNA from each isolate, and restriction fragments were ligated to double-stranded adaptors as described previously [10]. PCR was performed in a final volume of $25 \mu \mathrm{l}$ with 2 pmol of EcoRI adaptorspecific primer $(E c o R I+0)$ labelled with 5-carboxyfluoroscein (FAM) and 5 pmol of Mse I adaptor-specific primer $(M s e \mathrm{I}+\mathrm{C})$. Amplified fragments were separated in a denaturing polyacrylamide $5 \%$ gel, on an $\mathrm{ABI}$ Prism 377 DNA automated sequencer as described previously [10]. The fragments were sized with the ABI software GeneScan 2.1 and peak height thresholds were set at 100. Electropherograms of fragment profiles were inspected visually for polymorphisms. The presence and absence of fragments were scored in a binary matrix and recorded as a text (tab delimited) file in Excel v.5.0 (Microsoft). Dice coefficients of similarity were computed and cluster analysis was performed by the unweighted paired group method with arithmetic averages (UPGMA; NEIGHBOR pro- gram of PHYLIP). The resulting tree was displayed by using the TREEVIEW program [13] with a percentage similarity cut-off of $93 \%$ as described previously [11].

PFGE was performed as described previously [6]. The relatedness between the macrorestriction patterns was analysed with the comparative quantification module (with the band matching tolerance and optimisation set at 3 and 5 points respectively) in GelCompar software (Applied Maths, Kortrijk, Belgium).

\section{Results}

\section{FAFLP and cluster analysis}

Each isolate had a unique FAFLP profile. Among the 50 FAFLP profiles, there were 125 distinct amplified fragments $(E c o \mathrm{RI}+0, M s e \mathrm{I}+\mathrm{C})$, ranging in size from 55 to $320 \mathrm{bp}$. Thirty-four amplified fragments were common to all the MRSA isolates; the remaining 91 fragments were polymorphic. The average number of fragments constituting the FAFLP profile of an MRSA genome was $c$. 70. A percentage similarity cut-off of 93\% was applied to the FAFLP UPGMA tree analysis of the binary matrix, revealing nine clusters of MRSA (Fig. 1). There were 10-31 fragment differences between any two FAFLP clusters; the number of fragment differences between isolates within a cluster varied from 1 to 10 .

The 15 MRSA isolates from England and Wales were distributed among five FAFLP clusters. Clusters 1-3 corresponded to epidemic phage types EMRSA-16, EMRSA-15 and EMRSA-3. Other EMRSA phage types fell into two separate clusters (4 and 5 of Fig. $1)$, as has been described recently [11]. Six Continental European isolates also fell into clusters 3, 4 and 5. Cluster 1 contained a reference strain (E16) and four clinical isolates (X57, Y35, Y36, Y39) belonging to the epidemic phage type EMRSA-16. Cluster 2 (26-31 amplified fragment differences from cluster 1) contained a reference strain (E15) and three clinical isolates (X59, Y30, Y32) belonging to epidemic phage type EMRSA-15. A reference strain (E3) and a clinical isolate (Y8) belonging to phage type EMRSA-3 formed a third cluster; this cluster also included the Polish MRSA isolate X1, and had 22-24 amplified fragment differences from cluster 2. Cluster 4 contained the reference strain of EMRSA-1 (E1), an English clinical isolate (Y58) previously unassigned to a phage type, an epidemic isolate from Hungary (X6) and two sporadic MRSA isolates from Switzerland (X83, X123). Cluster 5, with 14-16 amplified fragment differences from cluster 4, included the reference strains of UK epidemic phage types EMRSA-2 (E2) and EMRSA10 (E10), grouped with epidemic MRSA isolates from Germany (X17) and Switzerland (X110).

Four further FAFLP clusters were delimited; among them were distributed 18 Continental European MRSA 
Table 1. Strains examined in study

\begin{tabular}{|c|c|c|c|c|}
\hline Isolate no.* & Country of origin & FAFLP $\dagger$ & $\begin{array}{l}\text { Cluster } \\
\text { PFGE: }\end{array}$ & $\begin{array}{l}\text { Multiprimer } \\
\text { RAPD§ }\end{array}$ \\
\hline Y56 & England & - & ND & ND \\
\hline X9 & Germany & 8 & 4 & $2 b$ \\
\hline XF1 & France & 8 & - & $2 b$ \\
\hline $\mathrm{X} 30$ & Belgium & 8 & - & $2 \mathrm{~b}$ \\
\hline X24 & Belgium & 8 & 4 & $2 b$ \\
\hline $\mathrm{X} 40$ & Switzerland & 8 & 4 & $2 b$ \\
\hline X7 & Portugal & 8 & 4 & $2 b$ \\
\hline $\mathrm{X} 5$ & Portugal & 8 & 4 & $2 \mathrm{~b}$ \\
\hline E10 (NCTC 13137) & England & 5 & ND & ND \\
\hline X17 & Germany & 5 & 5 & $2 b$ \\
\hline E2 (NCTC 12232) & England & 5 & ND & ND \\
\hline X110 & Switzerland & 5 & 5 & $2 b$ \\
\hline X83 & Switzerland & 4 & - & $2 \mathrm{a}$ \\
\hline $\mathrm{X} 123$ & Switzerland & 4 & - & $2 \mathrm{a}$ \\
\hline Y58 & England & 4 & ND & ND \\
\hline E1 (NCTC 11939) & England & 4 & ND & ND \\
\hline X6 & Hungary & 4 & - & $2 \mathrm{a}$ \\
\hline $\mathrm{X} 18$ & Austria & 7 & - & $2 a$ \\
\hline $\mathrm{X} 3$ & Poland & 7 & - & $2 \mathrm{a}$ \\
\hline X78 & Switzerland & - & - & - \\
\hline XF11 & France & 6 & - & 1 \\
\hline X12 & Germany & 6 & 3 & 1 \\
\hline $\mathrm{X} 65$ & Switzerland & 6 & - & 1 \\
\hline X37 & Switzerland & 6 & 3 & 1 \\
\hline $\mathrm{X} 1$ & Poland & 3 & - & 1 \\
\hline Y8 & England & 3 & ND & ND \\
\hline E3 (NCTC 13130) & England & 3 & ND & ND \\
\hline X80 & Switzerland & - & - & - \\
\hline X114 & Switzerland & - & - & - \\
\hline X77 & Switzerland & - & 1 & 3 \\
\hline $\mathrm{X} 59$ & England & 2 & 1 & 3 \\
\hline Y32 & England & 2 & ND & ND \\
\hline Y30 & England & 2 & ND & ND \\
\hline E15 (NCTC 13142) & England & 2 & ND & ND \\
\hline X103 & Switzerland & - & - & - \\
\hline X84 & Switzerland & - & - & - \\
\hline $\mathrm{X} 118$ & Switzerland & - & - & 5 \\
\hline X108 & Switzerland & 9 & 2 & 5 \\
\hline X21 & Germany & 9 & 2 & 5 \\
\hline $\mathrm{X} 46$ & Canada & 9 & 2 & 5 \\
\hline X29 & Belgium & 9 & 2 & 5 \\
\hline X124 & Switzerland & 9 & 2 & 5 \\
\hline X33 & Switzerland & 9 & 2 & 5 \\
\hline Y39 & England & 1 & ND & ND \\
\hline Y35 & England & 1 & ND & ND \\
\hline Y36 & Wales & 1 & ND & ND \\
\hline E16 (NCTC 13143) & England & 1 & ND & ND \\
\hline X57 & England & 1 & 6 & 5 \\
\hline X76 & Switzerland & - & 6 & 4 \\
\hline X131 & Switzerland & - & - & 4 \\
\hline
\end{tabular}

- , not assigned to a cluster; ND, no data.

* $\mathrm{X}$ strains are described in more detail in reference [12]. ' $\mathrm{Y}$ ' isolates are clinical isolates and ' $\mathrm{E}$ ' strains are reference epidemic MRSA strains from England and Wales as described in reference [11].

$\uparrow$ FAFLP cluster number derived from Fig. 1.

tPFGE cluster number derived from Fig. 2 .

§Clustering defined with multiprimer RAPD-PCR as described previously [12].

isolates and the sole North American MRSA isolate in this study. Cluster 6 had 15-19 amplified fragment differences from cluster 3, and contained non-epidemic isolates from Switzerland (X37, X65) and epidemic strains from Germany (X12) and France (XF11). Cluster 7 had 12-18 amplified fragment differences from cluster 4 and contained epidemic isolates from Poland (X3) and Austria (X18). Epidemic isolates from Portugal (X5, X7), Germany (X9), France (XF1), Belgium (X24, X30) and a non-epidemic isolate from Switzerland (X40) fell into an eighth cluster with 10-
15 amplified fragment differences from cluster 7 . The ninth cluster ( $>25$ fragment differences from either cluster 1 or cluster 8) contained epidemic isolates from Switzerland (X33, X108, X124), Belgium (X29) and Germany (X21), as well as a Canadian MRSA strain (X46).

A number of isolates did not fall into the well-defined FAFLP clusters described above. They included nine non-epidemic MRSA isolates from Switzerland (X118, $\mathrm{X} 76, \mathrm{X} 131, \mathrm{X} 84, \mathrm{X} 103, \mathrm{X} 77, \mathrm{X} 80, \mathrm{X} 114, \mathrm{X} 78$ ) and 


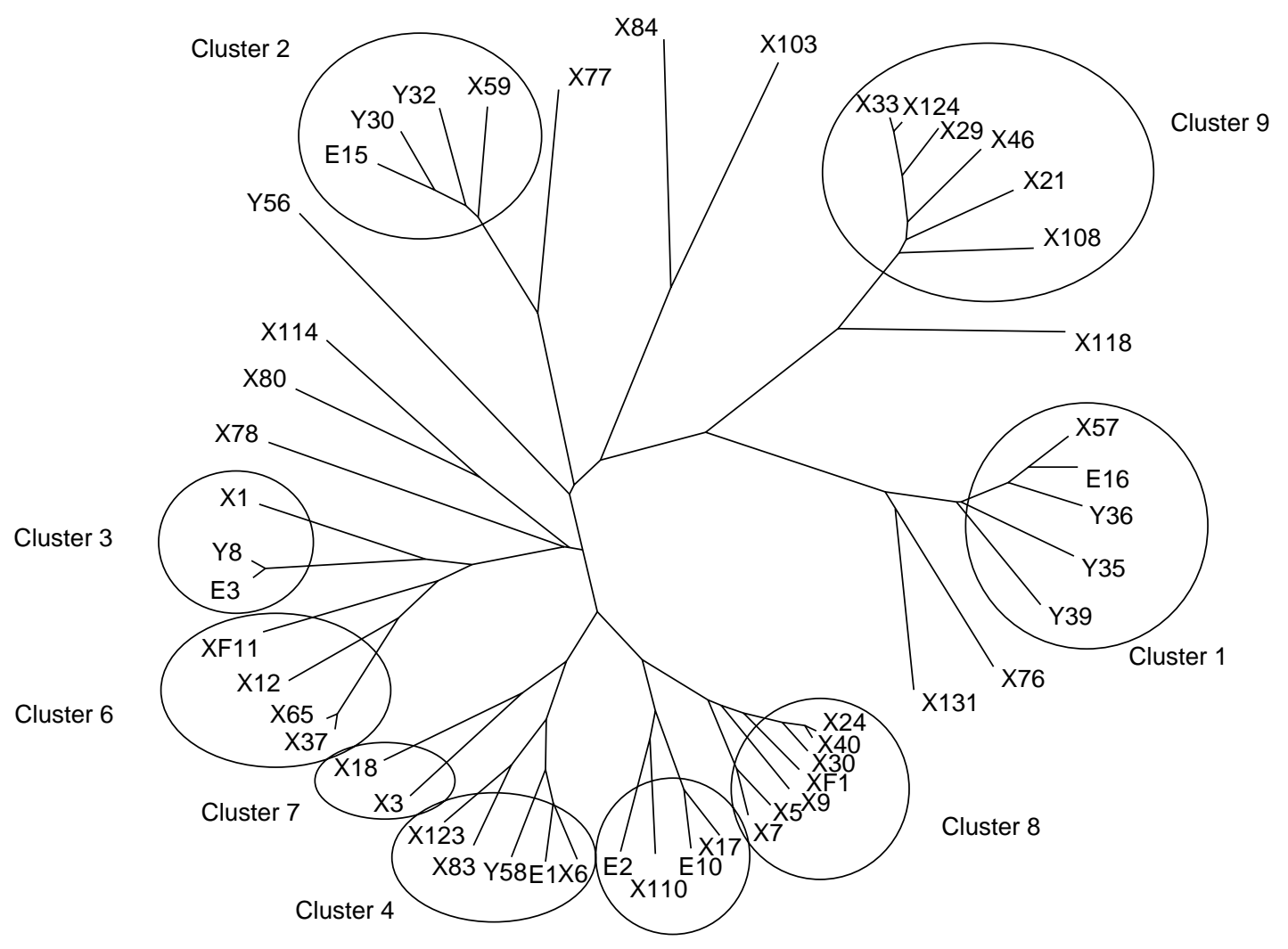

$10 \%$ divergence

Cluster 5

Fig. 1. UPGMA radial cluster tree derived from FAFLP data showing genetic relationships of MRSA. Each branch represents the unique FAFLP profile of a single isolate. See Table 1 for explanation of the labels. Horizontal scale bar represents $10 \%$ divergence.

one from England (Y56). Profiles of all these isolates differed by at least 10 amplified fragments from those delimited in the nine major clusters.

\section{PFGE analysis}

When macrorestriction and PFGE were applied, all isolates had unique profiles, with the exception of isolates X29 and X33 that displayed profiles identical to each other. A total of 63 distinct fragments was observed, which, in accordance with the methodology, we were not able to size precisely. The number of fragments constituting a PFGE profile varied from 14 to 19 (Fig. 2).

When a cut-off of $c .65 \%$ was used for analysis of the macrorestriction PFGE data, six clusters were found, broadly confirming the FAFLP results (Fig. 2). The lower percentage cut-off for PFGE data compared with FAFLP data reflects the considerably reduced number of data points in these profiles. Certain isolates not assigned to a cluster by FAFLP were clustered by PFGE. For instance, isolate X77 had a PFGE pattern related to isolate X59 (six band differences) and they were classed in the same PFGE cluster, but were not clustered by FAFLP (see above). Similar considerations also apply to isolates X76 and X57. Conversely, isolates XF1, XF11, X3, X30, X65, X83 and X123 clustered (although not identical) with other isolates by FAFLP, as also by RAPD-PCR [5], were distinct by macrorestriction PFGE.

\section{Discussion}

In this study, FAFLP defined nine clone complexes among European isolates. Two of these (clusters 1 and 2) correspond to EMRSA-16 and EMRSA-15, the most prevalent phage types currently found in England and Wales. FAFLP detected differences between all isolates and strains in these two tight clusters. There were a further seven FAFLP-defined clone complexes, as well as 10 'free-standing' isolates, which could have the potential to give rise to newly emerging epidemic strains. Three FAFLP clusters (clusters $4,6,8$ in Fig. 1) included isolates not associated with epidemic spread in a community. High-resolution FAFLP genotyping should assist in mapping the short-term evolution and dissemination of MRSA clones. Its high discriminatory power suggests that it may be particularly useful for resolving issues in local infection control. As it can display putative genetic relationships between current and even potential epidemic strains, it may be used to generate a practical model of the MRSA epidemic, both in local and in global terms. 

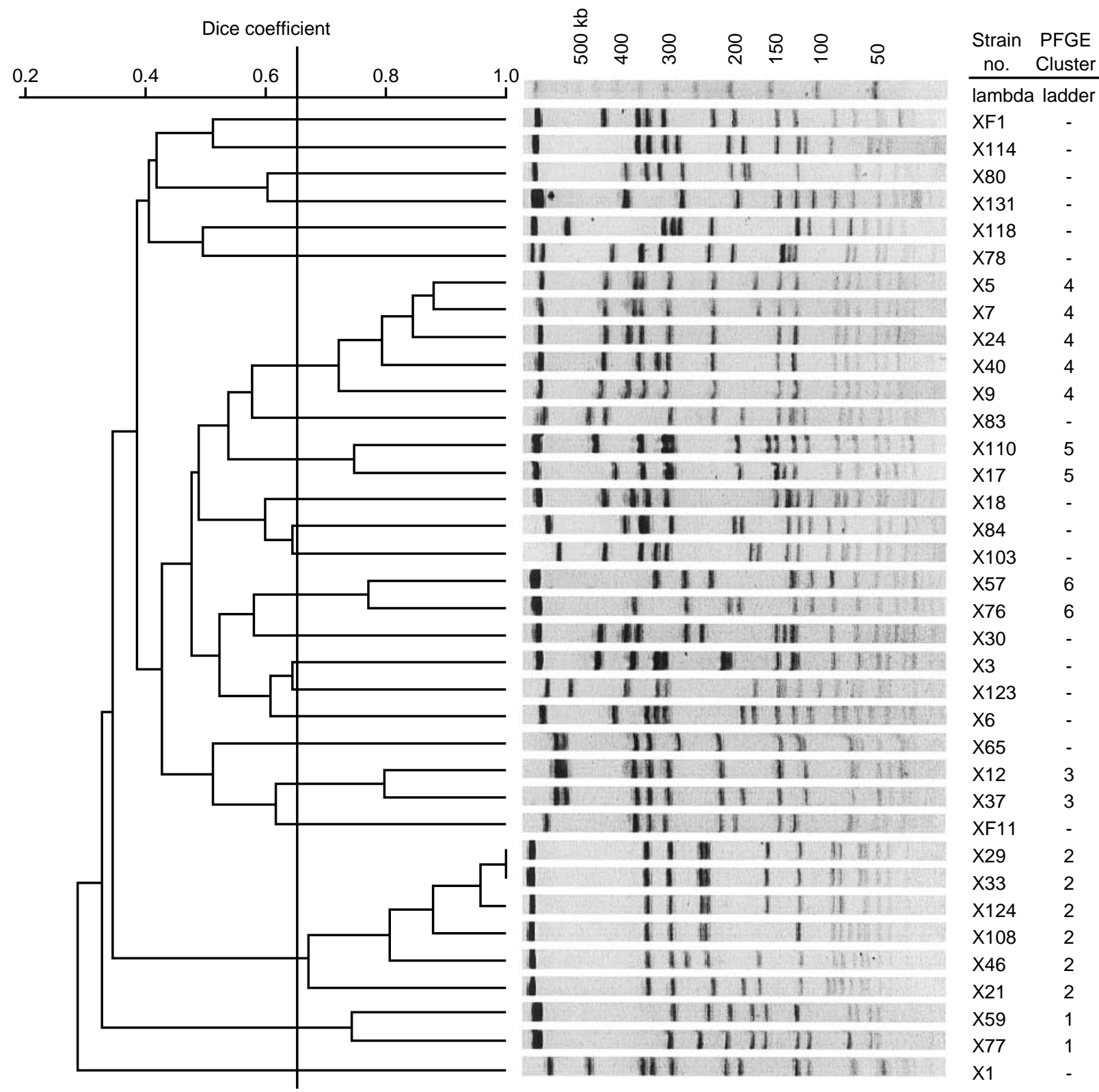

Fig. 2. UPGMA dendrogram derived from PFGE data and PFGE banding patterns. Each branch represents the unique macrorestriction profile of a single isolate.

A valid epidemiological typing system should exhibit high discriminatory power, be reproducible, easy to use, and capable of typing all isolates [14]. FAFLP analysis fulfils these criteria, exhibiting particular advantages with respect to reproducibility and discriminatory power [10,15-18]. While PFGE exhibits most of these criteria for many organisms, and has been applied with notable success in the PulseNet system for national epidemiological typing of $E$. coli O157 in the USA [7], it had lower discriminatory power than FAFLP in the present study of MRSA, and poorer reproducibility in an earlier study [10]. The ease-of-use of FAFLP analysis is likely to be further improved by robotics, automated sample loading and adaptation to multi-channel capillary sequencers. This high-resolution genotyping technique has now been applied successfully to the definition of epidemiological clonality in a range of other important bacterial pathogens including E. coli [15], Mycobacterium tuberculosis [18], Streptococcus pyogenes [16, 17], Neisseria meningitidis and Salmonella enterica serotype Enteritidis (unpublished observations). All these studies are characterised by unusually high reproducibility and discriminatory power. All polymorphic amplified fragments, which act as anonymous markers of genotype, were sized to within one base pair, an important and novel feature in the definition of a clone. Furthermore, experiments in this laboratory show that significant amplified fragments can readily be cloned for nucleotide sequencing. Although there is no published genome sequence for $S$. aureus at the time of writing, in the case of E. coli and M. tuberculosis, FAFLP analysis was modelled on, and relatable to, the published genome sequences. There are compelling theoretical reasons to believe that FAFLP can act as a bridge between the evolving database of whole 
bacterial genome sequences and the requirements of contemporary epidemiology.

Special thanks for provision of MRSA to B. Willey, H. de Lencaste, I Sanches, J. Etienne, M. Struelens, T. Berendt, I. Bowler, W. Hryniewicz, K. Trzinski, W. Witte, G. O’Neill and B. Cookson.

\section{References}

1. El Solh N, Casetta M, Aubert S, Morvan A, Allignet J. Typing of methicillin-resistant Staphylococcus aureus (MRSA) based on genome analysis. In: Brun-Buisson C, Casewell M, El Solh N, Régnier B (eds) Methicillin resistant staphylococci. Paris, Flammarion Médecine-Sciences. 1995: 46-64.

2. Anonymous. Methicillin-resistant Staphylococcus aureus. CDR Wkly Commun Dis Rep 1998; 8: 371-372.

3. Blair JE, Williams REO. Phage typing of staphylococci. Bull World Health Organ 1961; 24: 771-784.

4. Bannerman TL, Hancock GA, Tenover FC, Miller JM. Pulsedfield gel electrophoresis as a replacement for bacteriophage typing of Staphylococcus aureus. J Clin Microbiol 1995; 33: $551-555$.

5. Report of a Combined Working Party of the British Society for Antimicrobial Chemotherapy, the Hospital Infection Society and the Infection Control Nurses Association. Revised guidelines for the control of methicillin-resistant Staphylococcus aureus infection in hospitals. J Hosp Infect 1998; 39: 253-290.

6. Blanc DS, Petignat C, Moreillon P et al. Unusual spread of a penicillin-susceptible methicillin-resistant Staphylococcus aureus clone in a geographic area of low incidence. Clin Infect Dis 1999; 29: 1512-1518.

7. Strockbine NA, Wells JG, Bopp LA, Barrett TG. Overview of detection and subtyping methods. In: Kaper JB, O'Brien AD (eds) Escherichia coli O157:H7 and other Shiga toxinproducing E. coli strains. Washington, DC, ASM. 1998: 331-356.

8. van Belkum A, van Leeuwen W, Kaufmann ME et al. Assessment of resolution and intercenter reproducibility of results of genotyping Staphylococcus aureus by pulsed-field gel electrophoresis of SmaI macrorestriction fragments: a multicenter study. J Clin Microbiol 1998; 36: 1653-1659.

9. Iandolo JJ, Bannantine JP, Stewart GC. Genetic and physical map of the chromosome of Staphylococcus aureus. In: Crossley KB, Archer GL (eds) The staphylococci in human disease. New York: Churchill Livingstone. 1997: 39-53.

10. Grady R, Desai M, O’Neill G, Cookson B, Stanley J. Genotyping of epidemic methicillin-resistant Staphylococcus aureus phage type 15 isolates by fluorescent amplifiedfragment length polymorphism analysis. $J$ Clin Microbiol 1999; 37: 3198-3203.

11. Grady R, O'Neill G, Cookson B, Stanley J. Fluorescent amplified-fragment length polymorphism analysis of the MRSA epidemic. FEMS Microbiol Lett 2000; 187: 27-30.

12. Blanc DS, Banuls A-L, Hauser PM, Moreillon P, Tibayrenc M, and the Swiss MRSA Group 2000. Methicillin-resistant Staphylococcus aureus: phylogenetic relatedness between European epidemic clones and Swiss sporadic strains. Microb Drug Res 2000; 6: (in press).

13. Page RDM. Treeview: an application to display phylogenetic trees on personal computers. Comput Appl Biosci 1996; 12: $357-358$.

14. Maslow JN, Mulligan ME, Arbeit RD. Molecular epidemiology: application of contemporary techniques to the typing of microorganisms. Clin Infect Dis 1993; 17: 153-164.

15. Arnold C, Metherell L, Willshaw G, Maggs A, Stanley J. Predictive fluorescent amplified-fragment length polymorphism analysis of Escherichia coli: high-resolution typing method with phylogenetic significance. J Clin Microbiol 1999; 37: 1274-1279.

16. Desai M, Tanna A, Wall R, Efstratiou A, George R, Stanley J. Fluorescent amplified-fragment length polymorphism analysis of an outbreak of group A streptococcal invasive disease. J Clin Microbiol 1998; 36: 3133-3137.

17. Desai M, Efstratiou A, George R, Stanley J. High-resolution genotyping of Streptococcus pyogenes serotype M1 isolates by fluorescent amplified-fragment length polymorphism analysis. J Clin Microbiol 1999; 37: 1948-1952.

18. Goulding JN, Stanley J, Saunders N, Arnold C. Genomesequence-based fluorescent amplified-fragment length polymorphism analysis of Mycobacterium tuberculosis. J Clin Microbiol 2000; 38: 1121-1126. 